\title{
Erratum to: Long-term liposteroid therapy for idiopathic pulmonary hemosiderosis
}

\author{
Takehiko Doi $^{1} \cdot$ Shouichi Ohga ${ }^{1,2} \cdot$ Masataka Ishimura $^{1} \cdot$ Hidetoshi Takada $^{1}$. \\ Kanako Ishii $^{1}$ - Kenji Ihara ${ }^{1} \cdot$ Hideyuki Nagai $^{3}$. Toshiro Hara ${ }^{1}$
}

Published online: 30 September 2015

(C) Springer-Verlag Berlin Heidelberg 2015

Erratum to: Eur J Pediatr (2013) 172:1475-1481

DOI 10.1007/s00431-013-2065-9

The errata are noted below.

\begin{abstract}
The sentence, "Liposteroid was intravenously infused at 0.8 $\mathrm{mg} / \mathrm{kg} /$ day for three consecutive days at the time of acute bleeding." was corrected to "Liposteroid was intravenously infused at 0.05-0.08 $\mathrm{mg}$ (of dexamethasone) $/ \mathrm{kg} /$ day for three consecutive days at the time of acute bleeding."
\end{abstract}

\section{Page 1476, Methods, Liposteroid therapy, line 4-5}

The sentence, "It was intravenously infused at $0.8 \mathrm{mg} / \mathrm{kg} / \mathrm{day}$ for three consecutive days at the time of acute bleeding, after the obtainment of informed consent from parents." was corrected to "It was intravenously infused at $0.05-0.08 \mathrm{mg}$ (of dexamethasone) $/ \mathrm{kg} /$ day for three consecutive days at the time of acute bleeding, after the obtainment of informed consent from parents."

The online version of the original article can be found at http://dx.doi.org/ 10.1007/s00431-013-2065-9.

Shouichi Ohga

ohgas@pediatr.med.kyushu-u.ac.jp

1 Department of Pediatrics, Graduate School of Medical Sciences,

Kyushu University, 3-1-1 Maidashi, Higashi-ku, Fukuoka 812-8582, Japan

2 Department of Perinatal and Pediatric Medicine, Graduate School of Medical Sciences, Kyushu University, 3-1-1 Maidashi,

Higashi-ku, Fukuoka 812-8582, Japan

3 Division of Pediatrics, Kyoto Yamashiro General Medical Center, 27-1 Ikeda, Kizu, Kizukawa, Kyoto 619-0214, Japan 